\title{
The Design of Thermal-Insulating Mouse Pad Based on the Effect of Hand Temperature
}

\author{
Kristiana Asih Damayanti ${ }^{1,}$ a dan Shierli Siemadinata ${ }^{2, b}$ \\ 1,2 Industrial Engineering Department, \\ Parahyangan Catholic University, Bandung, Indonesia \\ akrist@unpar.ac.id and bshierli_siem@yahoo.com
}

Keywords: Thermal-Insulating Mouse Pad, hand temperature, carpal tunnel syndrome.

\begin{abstract}
Carpal Tunnel Syndrome (CTS) is the injury as the result of compression of the median nerve by the swollen muscle and soft tissues at carpal tunnel. This condition is causing pain and making the nerve's signal transmissions are slower. The muscle and soft tissues are swollen because of repeated movements and overuse so they don't have enough times for recovery. The muscle and tissues also press the blood flow which supplies oxygen and rich nutrient for them and make them swell. CTS will be worse if the tunnel is smaller because of terrible wrist's posture, and if the blood flow is slower which because of cold hand. The symptoms of CTS are pain, tingling, cold, and weak hand. Therapy and surgery can not totally heal this injury so it is much better to prevent it.

For reducing the risk of CTS, some actions can be done, for example making the hands always in a normal temperature or warm so the velocity of blood flow will be stable. Today, there is a product called fingerless gloves to keep hands warm but they are not really convenient so in this research another product is designed, which is thermal-insulating mouse pad.

In this study, an experiment is designed to prove that thermal-insulating mouse pad is working effectively. The experiment is done at climatic chamber with 40 students as respondents. There is one single factor of interest which has three levels. The treatments are the condition with no mouse pad, the condition using custom mouse pad, and the condition using thermal-insulating mouse pad. Data of experiment is collected by filling the questionnaire and measuring temperature of forearm, wrist, and finger. The experiment result shows that thermal-insulating mouse pad can keep hands warm when working with computer.
\end{abstract}

\section{Introduction}

Currently almost all aspects of human life have use of technology support and computer equipment, both in the world of work, education, and entertainment. For the future, human interaction with computer devices is expected to increase both the frequency and duration. In addition to providing many benefits such as ease, speed, and data processing, it turns out the use of computers can also be detrimental to human i.e. human health problem like the Carpal Tunnel Syndrome (CTS). CTS is a lot of happen on the user's computer so that work performance not optimal and also increase the number of workers' absent.

CTS is carpal injuries in the channel on the wrist where the median nerve in the channel is suppressed by muscle and other soft tissues are swollen, because of the excessive use of hand muscles. This situation will get worse if the space due to the narrower channels carpal wrist posture is not good, and the slower blood flow due to cold temperatures hand. The symptoms are usually felt by people suffering from the onset of CTS pain in hands, thumbs become numb; hands become cold, tingling, weakness and stiffness. Various healing actions can be performed, such as ultrasonic treatment or perform surgery, but such actions have not been proven to cure or totally recovered to the original state. Therefore, it is better than cure if a computer user perform preventive actions, one of which is to keep hands warm temperatures

One of the precautions that can be done to reduce the risk of CTS is to keep hands warm so blood flow does not become slow. Slower blood flow resulting in the muscles and soft tissues like tendons and ligaments are deprived of oxygen and nutrients to become swollen. Swelling of the 
muscles and soft tissues will be pressing the blood vessels and the median nerve. The product that is currently circulating to keep the temperature of the hands is fingerless gloves. But this product has some weaknesses, which is less practical, uncomfortable, and cannot fix the hand posture. When using a computer, users often interact with the mouse, so the product that will be designed in this study is a mouse pad, especially the thermal-insulating mouse pad.

Instead the function of thermal-insulating mouse pad is often used, is still to be seen whether its use had a significant influence on the temperature of the arm, wrist, and fingers or not, while working with computer users. To prove it, it is necessary to do an experiment. Besides viewing aspects of the benefits, convenience aspect of the user when use these products who does not interfere the performance also important. Therefore, it is required range of reference and information derived from the respondents about their opinions related to the design of the product.

Based on the identification of problems, the object of the study is to:

a. Identification the influence of a thermal-insulating mouse pad on temperature changes in the arms, wrists, and fingers when working with computers.

b. Design an ergonomic thermal-insulating mouse pad

\section{Methods}

The study was developed from the research that doing by Meijer [1] and conducted in laboratory by make an experiment. The experiment was carried out in climatic chamber. The design of thermal-insulating mouse pad is intended to reduce the risk of Carpal Tunnel Syndrome. The product design of thermal-insulating mouse pad does not take into account the financial aspect. The assumptions used in this study are the changes of body position of respondents did not affect the temperature measurement results and conditions at experimental room are the same with using of a thermal-insulating mouse pad.

Sampling technique which was used in this study was non-probability sampling, (purposive sampling). Research instruments were by makes experiment and questionnaires. Data which is collected from the measurement results processed and analyzed with statistical method. The result of data processing and analysis was used as a basis for designing a thermal-insulating mouse pad. Design results are analyzed and evaluated to determine strengths and weaknesses of the product.

1.1. Determination of experimental objective

Experiments aimed to determine the effect of treatment using a mouse pad type to temperature changes of the forearm, wrist, and fingers of respondents when working with computers. Respondents of this experiment was university students as many as 40 with a range of ages 20 to 23 years old. Sample or the respondent must meet the criteria and can represent the population, i.e. they are active computer users.

1.2. Determination of controllable factor

To improve the accuracy of experimental data is by minimizing the factors that are not desired [2], and then other factors that potentially affect the value of the dependent variable, namely the temperature of the hand, must be controlled. Factors that been controlled was 1.) Temperature of the space environment experiments, i.e. in accordance to Tichauers' research that the ambient temperature is controlled at a value of $25^{\circ} \mathrm{C}[3]$, 2.) Position of the body of respondents, which is adjusted with reference to posture ergonomics when using a computer, one of which is shoulders and wrists are not rose in a straight position so it not impede blood flow, 3.) Stress levels of respondents, because the level of stress can cause the temperature of hand become cool. Control stress level is done by giving the task with the same level of difficulty for all respondents in the absence of performance targets.

1.3. Preparing the site and experimental equipment

Experiments conducted in a climatic chamber and the equipment used is an air conditioner (AC), room thermometer, work desk, adjustable chair, laptop, mouse, mouse pad, a thermal machine, thermometer, camera, stop watch, form an example of text, and the questionnaire, all the equipments is shown in the Figure 1. Questionnaires are useful for obtaining qualitative 
data. Fill in the questionnaire in general is about the personal data of respondents, the questions relating to the habits of respondents related to the use mouse and mouse pad products, CTS symptoms are felt, the choice of the mouse pad, and their opinions related to the experiments that have been made.

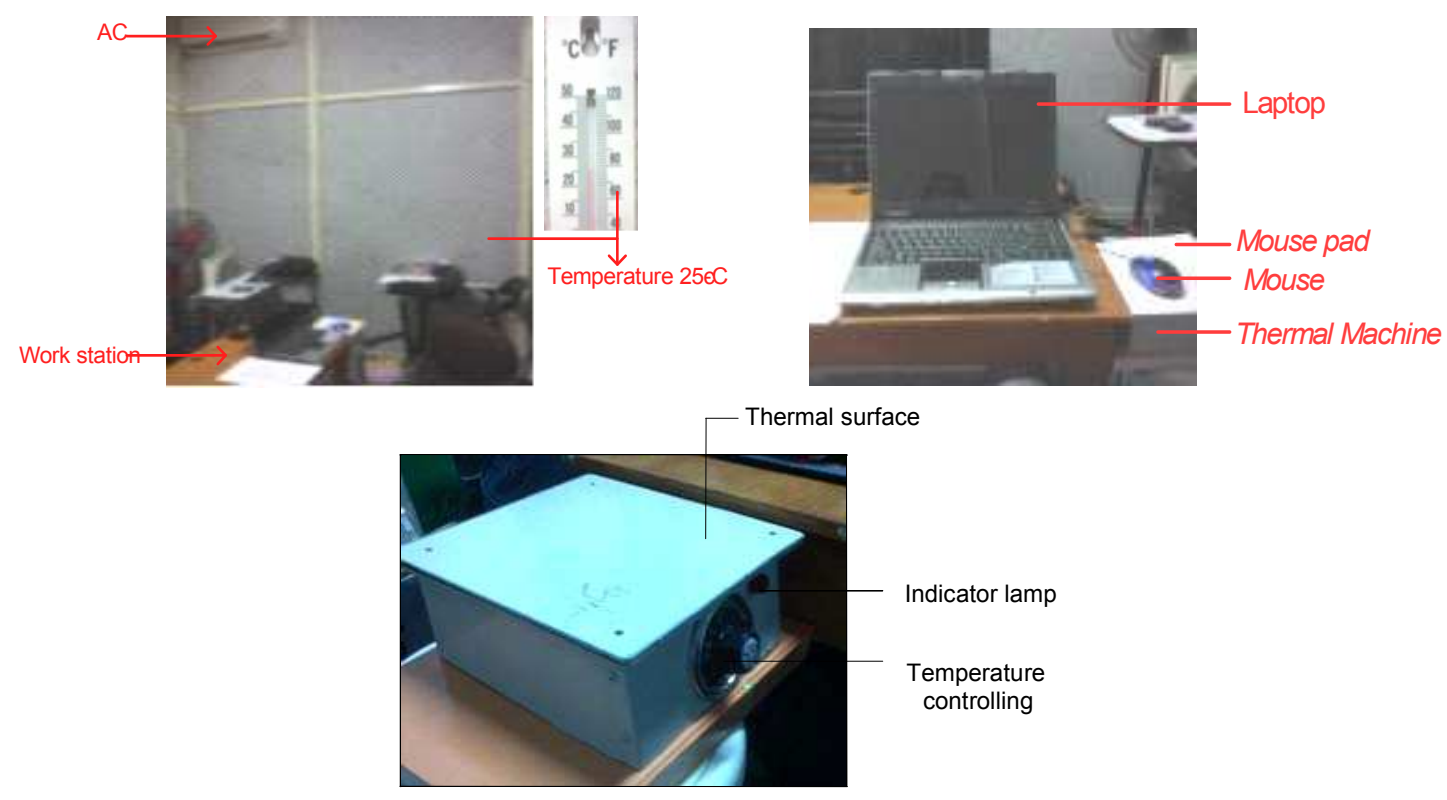

Figure 1. Experiment tools

Experimental procedure begins with the adaptation process in which respondent can adapt to environmental temperature. At this stage, the temperature of the hands of the respondents was measured at intervals of every 3 minutes. In the experimental stage, to 3 conditions or treatment that respondents did not use a mouse pad, respondents use a mouse pad, and the respondents using a thermal-insulating mouse pad. On each condition, the respondent must work with computers where there are two kinds of tasks that must be done, namely a mouse task and mouse combination task. On mouse task, respondents was using the mouse only for works, while at the combination task, the respondent must used the mouse and keyboard interchangeably. Task simulated mouse activity is done by ask the respondent to play a game while the combination task is done by ask the respondent type text and underline text with the mouse. Each task performed for 20 minutes. At this stage, the temperature measurements of the forearm, wrist, and fingers performed every 5-minute intervals. After the experiment, respondents then completed a questionnaire experiment.

The experiment data collected, and then the subsequent data is processed. Temperature measurement data were statistically processed using ANOVA test Completely Randomized Design with a value of $\alpha=5 \%$. Factor was use consisting of 3 treatments. In ANOVA, then there are some assumptions that must be fulfilled that are: normally distributed data, residual data have normally distributed and independent, constant variance data. Conclusions from the results of the ANOVA testing treatment differences significantly influence the temperature difference of the forearm, wrist, and fingers as well as a mouse task and task combination. In addition to statistically tested, the data were plotted in graphical form to analyze the movement of the temperature. It appears that when using a thermal-insulating mouse pad, the temperature of the hand is more stable and warmer than usual when using the mouse pad and when not to use a mouse pad.

\section{Results}

Based on the results of data processing and analysis, the design products of thermalinsulating mouse pad can be seen at figure 2. The design of products is looking like figure 2 below. Square shape is equipped with attributes wrist rest, heating elements, thermostats, temperature control knob, indicator lights, and a USB cable to take electrical energy from the computer. 
Materials which is used: soft nylon cloth at the top, non-skid rubber on the base, a heating element consisting of plates of iron and copper coils, aluminum foil, electrical insulators, and silicone gel on the inside of the wrist rest.

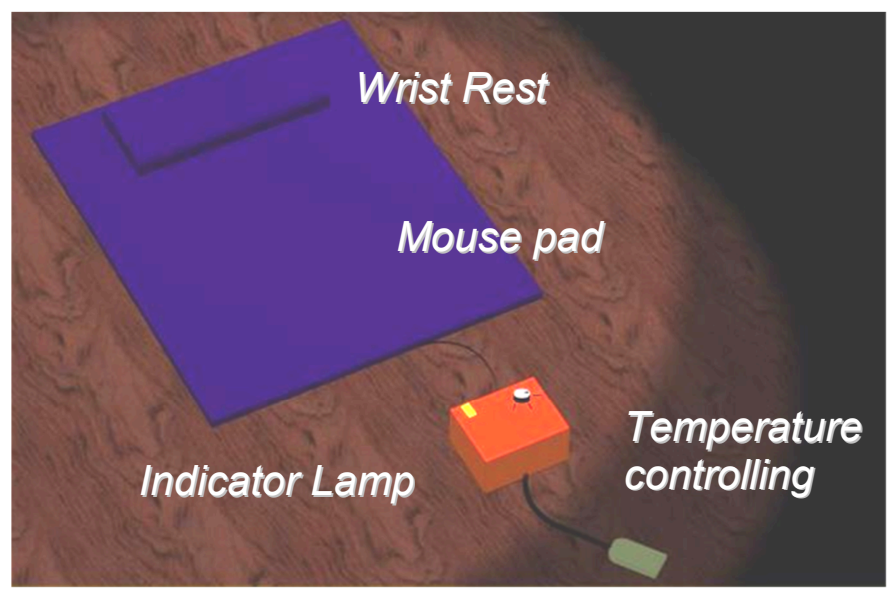

Figure 2. The design of Thermal-Insulating Mouse Pad

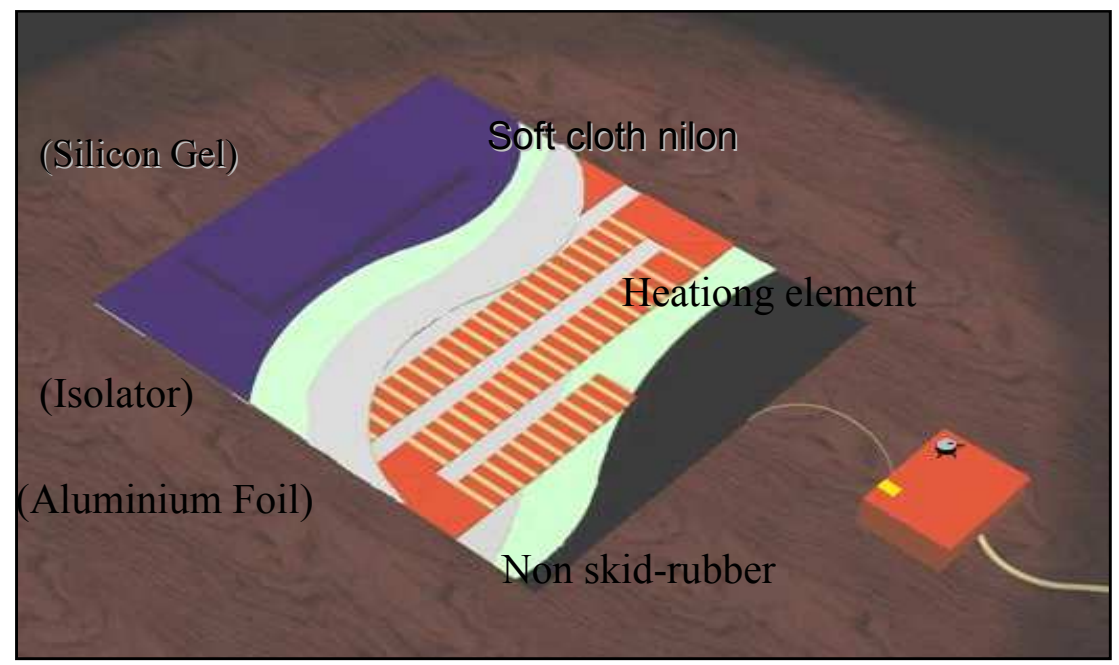

Figure 3. Material of Thermal-Insulating Mouse Pad

The design (at Figure 2 and 3 ) has positive and negative factors. The positive is that it can serve to keep the hands warm temperature, sufficient size, that is not too big or too small, has a wrist rest that is wider so that the wrist can move more freely, safe from electrical, and temperature can be set. While the negative is this product requires electrical energy, inflexible, and their placement is depend on cable length.

\section{Conclusion}

Based on the results of research has been done, the following is the conclusion of the study:

a. Based on experimental results, the use of thermal-insulating mouse pad provides a significant influence on temperature changes of the forearm, wrist, and fingers of respondents currently use a computer. The temperature of the arm and hand of respondents currently use a thermal-insulating mouse pad is more stable than when the respondents use a mouse pad or mouse pad does not use either the mouse when the task or task combination. Testing the significance of the influence of treatment carried out with the statistical test Analysis of Variance Completely Randomized Design.

b. Thermal-insulating mouse pad is designed to make the hand temperature is maintained at a normal temperature so as to make blood flow smoothly and keep the muscles and soft tissues in the carpal channel does not swell. Thus it can reduce the risk of Carpal Tunnel Syndrome. Performed 
included the design of the form factor, size, components, and materials used, so design goals can be achieved that these products can function effectively, convenient and safe to use, healthy for its users, and efficient. Here are the specifications of the thermal-insulating mouse pad that is designed:

1) Mouse pad is rectangular-shaped cross section with vertical orientation and has a wrist rest assembly attribute in the form of flat rectangular cross section.

2) The dimension of Mouse pad is $24 \mathrm{~cm} \times 20 \mathrm{~cm}$ while the wrist rest assembly is $12 \mathrm{~cm} \times 5$ $\mathrm{cm}$. The maximum thickness of the mouse pad is $1.5 \mathrm{~cm}$

3) Components of the mouse pad is:

a. The upper surface mouse pad with a soft cloth material is nylon fabric b. The surface below of the mouse pad is made by non-skid rubber material. c. The middle layer which is a heating element by using induction heating technique which consists of plate iron, copper coils, and thermostats as temperature control. For security, the heating element is coated with aluminum foil and rubber electrical insulators.

d. Wrist rest assembly using silicone gel material.

e. USB cable as a power source.

f. Indicator lights.

\section{References}

[1] Meijer, Eline M. 2006. Effects of a thermal-insulating mouse pad on temperature of forearm and hand during computer task. Universiteit of Amsterdam ,The Netherlands.

[2] Hicks, Charles R. 1982 . Fundamental Concepts in the Design of Experiments. $3^{\text {rd }}$ ed. HoltSaunders International Edition. New York.

[3] Pulat, Mustafa B. 1992, Fundamental of Industrial Ergonomics. Waveland Press, Inc. Illinois, United States of America.

[4] Zontak, Alla,et al. 1997. Dynamic Thermography: Analysis of Hand Temperature During Exercise. Haifa, Israel. 